Ann. Sc. forest., 1980, 37 (2), 159-172.

\title{
Nutrition minérale et production des peupliers « Robusta » et « I-214 » en populiculture traditionnelle dans le nord du bassin parisien
}

\author{
J. GARBAYE \\ avec la collaboration technique de G. COURRIER et Louisette MASAR \\ Station de Recherches sur les Sols forestiers ef la Fertilisation \\ Centre nationol de Recherches forestières, I.N.R.A., \\ Champenoux 54280 Seichamps
}

\begin{abstract}
Résumé
Les relations entre les teneurs dans le sol et les feuilles de $\mathrm{N}, \mathrm{P}, \mathrm{S}, \mathrm{K}, \mathrm{Ca}, \mathrm{Mg}$ et $\mathrm{Mn}$ et la production des peupliers Populus $\times$ euramericana, cv. "Robusta " et " $1-214$ ", ont été étudiées dans soixante six peupleraies du nord du bassin parisien, gérées de façon traditionnelle (sans fertilisation ni travail du sol).

Sur les sols hors vallée, sans nappe permanente, la production est liée avant tout à la présence de carbonate de calcium dès la surface du sol, qui entraîne une diminution de croissance accompagnée d'une réduction de l'absorption de manganèse.

Sur les sols alluviaux à nappe permanente, le premier facteur limitant la production est le régime hydrique (alimentation en eau insuffisante ou exès d'hydromorphie), déterminé par la profondeur d'apparition de la réduction permanente et la profondeur d'apparition de la réduction temporaire.

Lorsque l'alimentation en eau est proche de l'optimum, les disponibilités en azote et en phosphore du sol (horizon $A_{1}$ ) deviennent limitantes. La teneur en phosphore assimilable de l'horizon $A_{1}$ (méthode Duchaufour-Bonneau) est liée de façon très hautement significative à la teneur en phosphore des feuilles et à la production. Une production supérieure à $15 \mathrm{~m}^{3}$ par ha ef par an à 20 ans pour « Robusta » (volume total de la tige) nécessite 0,3 p. 1000 de $P_{2} O_{5}$ assimilable dans l'horizon $A_{1}$ et environ 0,3 p. 100 de $\mathrm{P}$ total dans les feuilles au sommet des arbres à la fin du mois d'août.
\end{abstract}

\section{Introduction}

Dans un travail précédent et dans cette même revue (Garbaye, 1979), nous avions éfudié l'influence du type de sol sur la production des peuplier Populus $\times$ euramericana, cv. « Robusta » et «|-214», en populiculture traditionnelle (c'est-à-dire sans fertilisation ni entretien prolongé du sol) dans le nord du Bassin parisien.

Il était apparu que la production dépendait avant tout de la présence ou de l'absence de calcaire dans le cas des sols hors vallées sans nappe permanente, et du régime et de la profondeur de la nappe dans le cas des sols alluviaux dans les vallées.

Le tableau 1 donne le détail des six ensembles de sols définis d'après ces critères simples, avec les productions correspondantes pour les deux clones. 


\section{TABLEAU 1}

Type de sol et production de «Robusta » ef « 1-214» en $\mathrm{M}^{3} /$ ha/an à 20 ans, volume fotal fin bout. (PRP : Profondeur d'apparition de la réduction permanente ; PRT : profondeur d'apparition de la réduction temporaire.) Cf. Garbaye, 1979.

Soil type and production of "Robusta " and « $\mathrm{I}-214$ » in $\mathrm{m}^{3} / \mathrm{ha} /$ year at 20 years, fotal volume up to the thin end (PRP : depth of the beginning of permanent reduction ; PRT : depth of the beginning of temporary reduction.) Cf. Carbaye, 1979.

\begin{tabular}{|c|c|c|c|c|c|c|c|}
\hline \multirow{2}{*}{\multicolumn{2}{|c|}{ Ensemble de sols }} & \multicolumn{3}{|c|}{ «Robusta» } & \multicolumn{3}{|c|}{ «|-214 » } \\
\hline & & Moyenne & $\begin{array}{l}\text { Valeurs } \\
\text { extrêmes } \\
\text { dans } \\
\text { l'échan- } \\
\text { tillon }\end{array}$ & $\begin{array}{l}\text { Ecart- } \\
\text { type }\end{array}$ & Moyenne & $\begin{array}{l}\text { Valeurs } \\
\text { extrêmes } \\
\text { dans } \\
\text { l'échan- } \\
\text { tillon }\end{array}$ & $\begin{array}{c}\text { Ecart- } \\
\text { type }\end{array}$ \\
\hline $\begin{array}{l}\text { Sols sans nap- } \\
\text { pe perma- } \\
\text { nente (hors } \\
\text { vallées) }\end{array}$ & $\left\{\begin{array}{l}\text { Ensemble } A \\
\text { (sans calcaire dès la surface } \\
\text { Ensemble } B \\
\text { (avec calcaire dès la surfa- } \\
\text { ce) } \ldots \ldots \ldots \ldots \ldots \ldots \ldots\end{array}\right.$ & 10,8 & $\begin{array}{l}9,5-13,8 \\
5,0-9,5\end{array}$ & 2,0 & 9,9 & $7,8-12,2$ & 1,9 \\
\hline $\begin{array}{l}\text { Sols avec } \\
\text { nappe per- } \\
\text { manente } \\
\text { (sols allu- } \\
\text { viaux en } \\
\text { fond de val- } \\
\text { lées) }\end{array}$ & 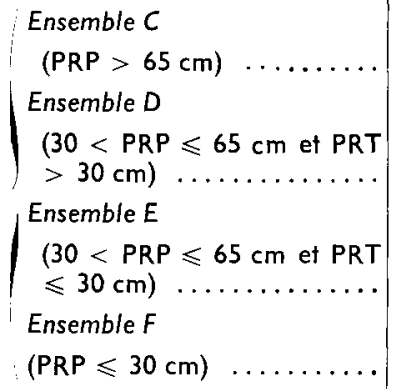 & 14,4 & $10,0-23,0$ & 1,7 & 14,5 & $\begin{array}{r}13,0-17,0 \\
7,4-11,5\end{array}$ & 1,5 \\
\hline
\end{tabular}

Ce classement est loin d'expliquer toutes les différences de production observées : la variabilité interne de chaque ensemble de sols reste grande, et ne permet de prévoir la production que dans des limites assez larges. Parmi les causes de cette variabilité, on peut évoquer les variations climatiques dans la zone éfudiée, les techniques de plantation, la nature de la végétation concurrente, et les caractéristiques chimiques du sol qui conditionnent la nutrition minérale des arbres. C'est ce dernier point que nous étudierons ici.

\section{Matériel et méthodes}

L'échantillon est le même que pour la première étude, à laquelle nous renvoyons pour le détail. II comprend trente neuf placettes de « Robusta » et vingt sept placettes de «|-214» distribuées dans le nord du Bassin parisien. Dans chaque placette, cinq arbres voisins ont fait l'objet d'analyses foliaires. Les feuilles ont été prélevées au fusil dans le tiers supérieur de la couronne, entre le 25 ef le 28 août 1975 . Une étude 
préliminaire avait permis de déterminer les modalités optimales de prélèvement (Garbaye, 1972).

Les cinq échantillons provenant de chaque placette ont fait l'objet des déterminations suivantes, après séchage à $65^{\circ} \mathrm{C}$ et broyage :

- après minéralisation par l'eau oxygénée en milieu sulfurique : azote total par colorimétrie à l'autoanalyseur «technicon»;

- après minéralisation par l'eau oxygénée en milieu perchlorique : phosphore total par colorimétrie à l'autoanalyseur « Technicon », soufre total par turbidimétrie, et potassium, calcium, magnésium ef manganèse totaux par spectrophotométrie d'absorption ou d'émission.

Chaque horizon de sol a fait l'objet des analyses suivantes:

- carbone total (méthode Anne : attaque à chaud par le bichromate de potassium en milieu sulfurique);

- azote total (méthode Kjeldahl);

$-\mathrm{pH}$ (eau) ;

- cations échangeables par percolation à l'acétate d'ammonium à pH $7: \mathrm{Ca}$ (échantillons non calcaires seulement), $\mathrm{K}, \mathrm{Mg}$ et $\mathrm{Mn}$;

- manganèse réductible (percolation à l'acétate d'ammonium en présence d'un réducteur : le chlorhydrate d'hydroxylamine) (Coppenet, 1959);

- phosphore assimilable (méthode Duchaufour-Bonneau, 1969 avec double extraction acide et basique).

\section{Résultats et discussion \\ Valeurs moyennes et amplifude de variation des teneurs foliaires. \\ Différences entre placettes}

Le tableau 2 montre que l'amplitude de variation des valeurs moyennes par placette est grande pour tous les éléments ; elle est maximale pour le manganèse (de 32 à 347 p.p.m.). La faible variabilité intra-placette (entre les cinq arbres échantillonnés) fait que le $F$ inter-placettes est très hautement significatif dans tous les cas (risque de 1 p. 1000 ). Les éléments qui différencient le mieux les placettes globalement sont le manganèse et le magnésium, puis le soufre et le phosphore. Ceci est vrai pour les deux clones.

Les valeurs observées encadrent les valeurs généralement considérées comme optimales (Carbaye, 1972) : 2 à 2,5 p. 100 pour N, 0,2 à 0,5 p. 100 pour $P, 1,2$ à 1,5 p. 100 pour $K$. Le magnésium fait exception et présente des valeurs toujours supérieures à 0,19, alors que l'optimum est généralement placé à 0,15 p. 100 .

\section{Différences de nutrition entre ensemble de sols}

Le tableau 3 montre que, pour la plupart des éléments, la composition minérale des feuilles varie très peu, et de façon non significative, entre les six ensembles de sols précédemment définis. Ceci ne nous étonne pas, car ces ensembles ont avant tout été constitués en tenant compte du régime hydrique et non pas de la chimie des sols, 


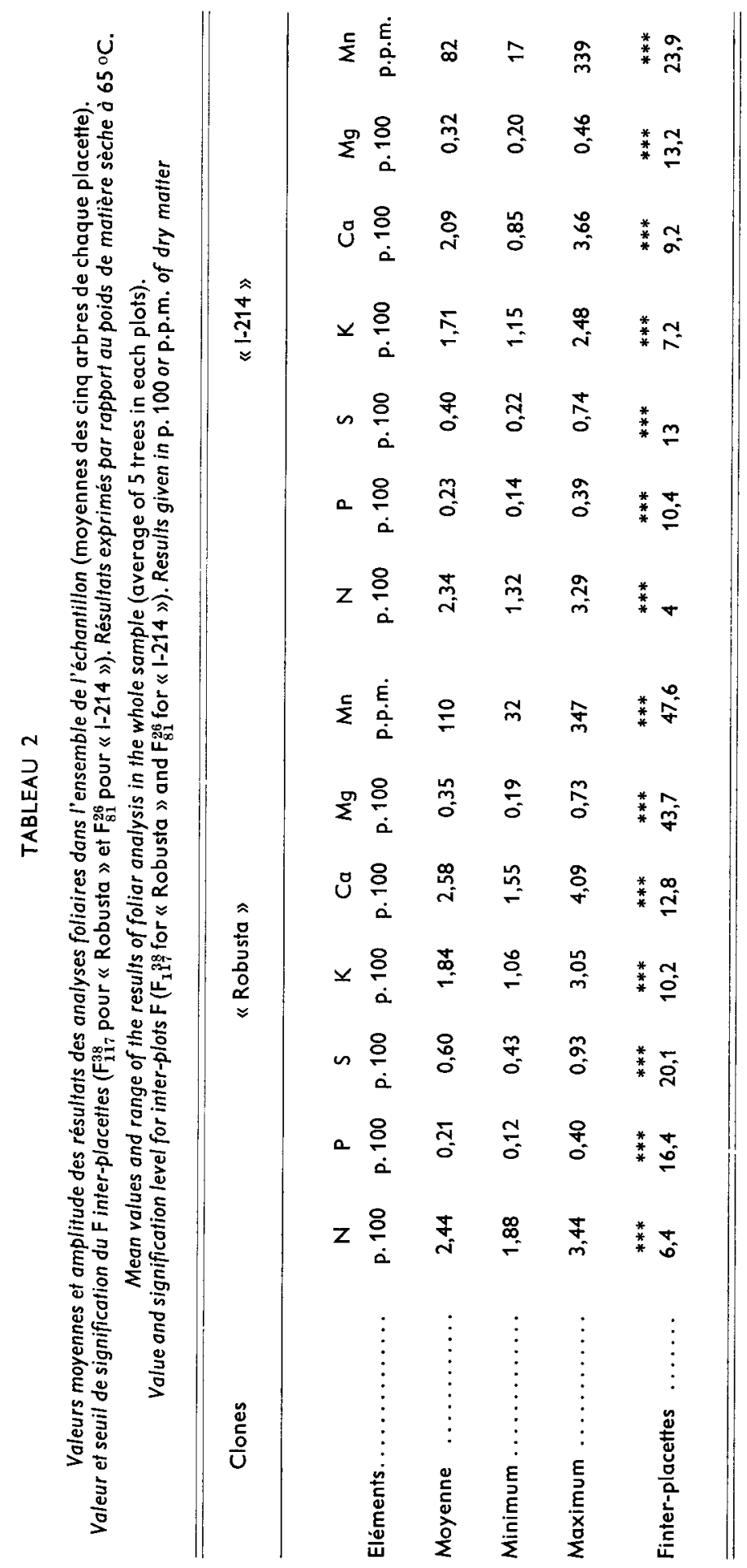




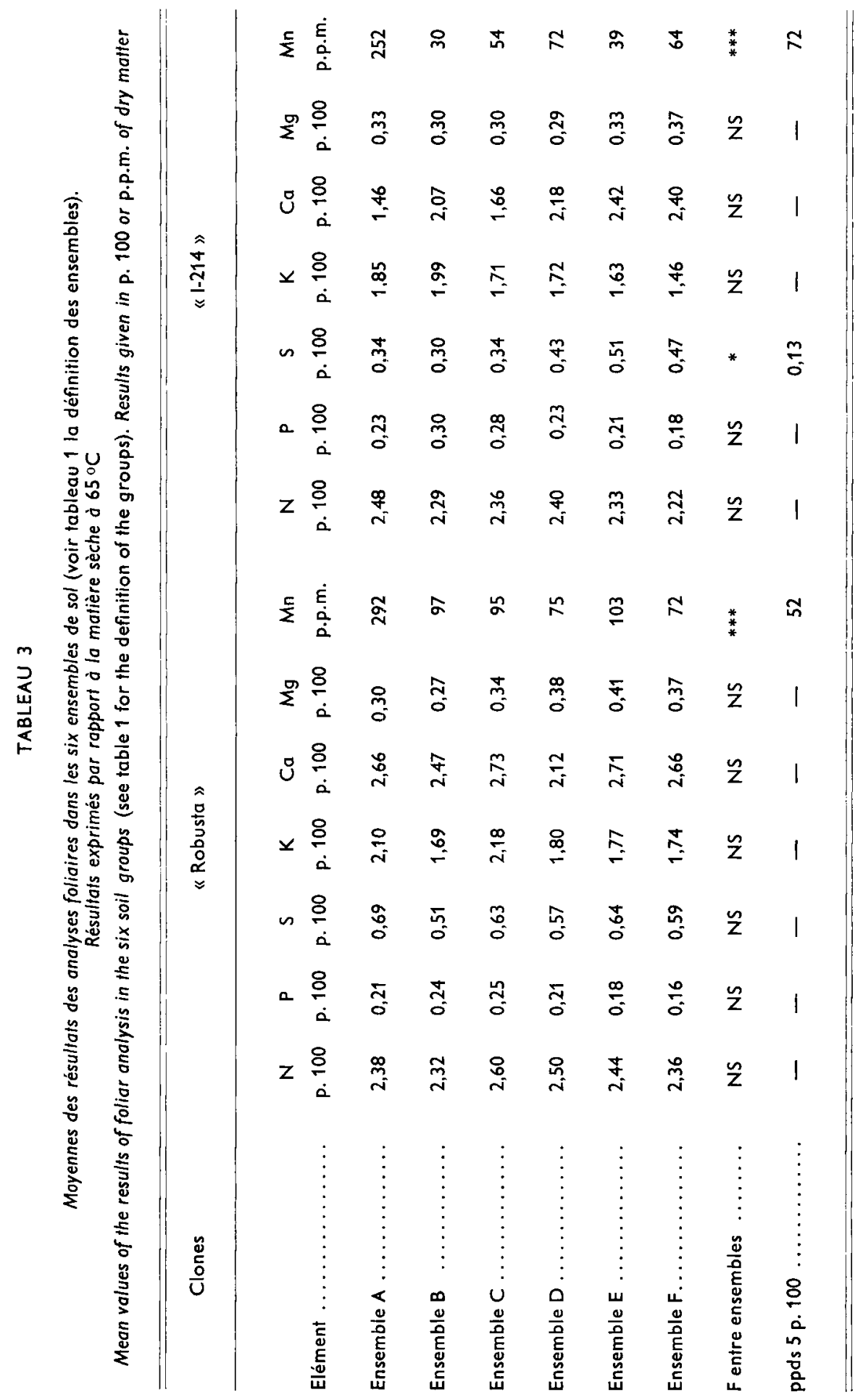


sauf pour les ensembles $A$ et $B$ qui ne diffèrent que par l'absence $(A)$ ou la présence $(B)$ de carbonate de calcium dans l'horizon $A_{1}$. Or, on voit qu'un élément qui fait exception, le manganèse, est environ trois fois plus abondant pour les deux clones dans l'ensemble $A$ (sols non calcaires) que dans les autres ensembles (sols toujours calcaires pour $B$, et presque toujours pour $C, D, E$ ef $F$ ).

Cette forte absorption de manganèse sur les sols décarbonatés a déjà été constatée par Le Tacon ef al. (1970) avec l'épicéa (Picea abies). Pour cette espèce, le phénomène était cependant beaucoup plus net, puisque le manganèse était huit fois plus abondant dans les aiguilles sur les sols décarbonatés que sur les sols carbonatés, ef il était accompagné de teneurs plus fortes en azote et plus faibles en calcium sur les sols décarbonatés, ce qui n'est pas le cas ici pour les peupliers.

Chez l'épicéa, type de l'essence sensible au calcaire, la production était supérieure sur les sols décarbonatés. Dans notre cas, on ne retrouve cette différence que pour «Robusta 》 (tabl. 1).

Les deux clones de peupliers considérés se comportent donc comme une espèce moyennement sensible au calcaire, «Robusta» étant plus sensible que «|-214». Son comportement est intermédiaire entre celui de l'épicéa (Le Tacon ef al., 1970) et celui du Pin noir d'Autriche (Le Tacon, 1974) espèce parfaitement tolérante au calcaire.

Le tableau montre que le soufre présente également des différences de teneurs foliaires entre ensembles de sols, mais chez « $1-214$ 》 seulement : les teneurs sont les plus élevées sur les sols où l'hydromorphie est la plus prononcée (ensembles $E$ ef $F$ ). Ce résultat sera discuté plus loin.

\section{Relations sol-nutrition-production}

Après avoir constaté que le découpage de l'échantillon en ensembles de sols définis par leur régime hydrique ne permettait pas de mettre en évidence d'éventuelles relations entre la nutrition minérale et la production (à l'exception du cas du manganèse sur les sols sans nappe permanente), nous avons procédé de façon différente, en calculant les coefficients de corrélation linéaire entre les teneurs foliaires, la production et éventuellement les teneurs de l'horizon $A_{1}$ du sol en éléments nutritifs (le tableau 4 donne la moyenne et l'amplitude des caractères chimiques de l'horizon $A_{1}$ dans les différents ensembles). Pour le phosphore assimilable ef le potassium échangeable, les calculs ont également été faits sur la quantité totale par hectare contenue dans les 50 premièrs $\mathrm{cm}$ du sol. Cette façon de faire n'apporte rien par rapport à la seule prise en compte des teneurs relatives dans l'horizon $A_{1}$ :

- sur l'ensemble de l'échantillon (les deux clones étant traités séparément), aucune relation n'apparaît ;

- sur les sols sans nappe permanente, aucune relation n'apparaît non plus ;

- sur l'ensemble des placetfes sur sols alluviaux à nappe permanente, une seule liaison est significative (risque de 1 p. 100) : $P$ feuilles $-\mathrm{P}_{2} \mathrm{O}_{5}$ assimilable dans l'horizon $A_{1}$, et ceci uniquement pour « Robusta ». Aucune liaison n'apparaît avec la production, ce qui suggère que le premier facteur limitant la croissance des peupliers est l'alimentation en eau, la nutrition minérale ne jouant qu'un rôle secondaire. Remarquons d'ailleurs que c'est le cas pour le frêne (Fraxinus excelsior) comme l'ont montré Lévy ef al. (1977) dans le nord-est de la France. Sur la base de cette hypothèse, nous avons divisé l'échantillon « sols alluviaux » en deux : d'un côté les ensembles de sols où le 


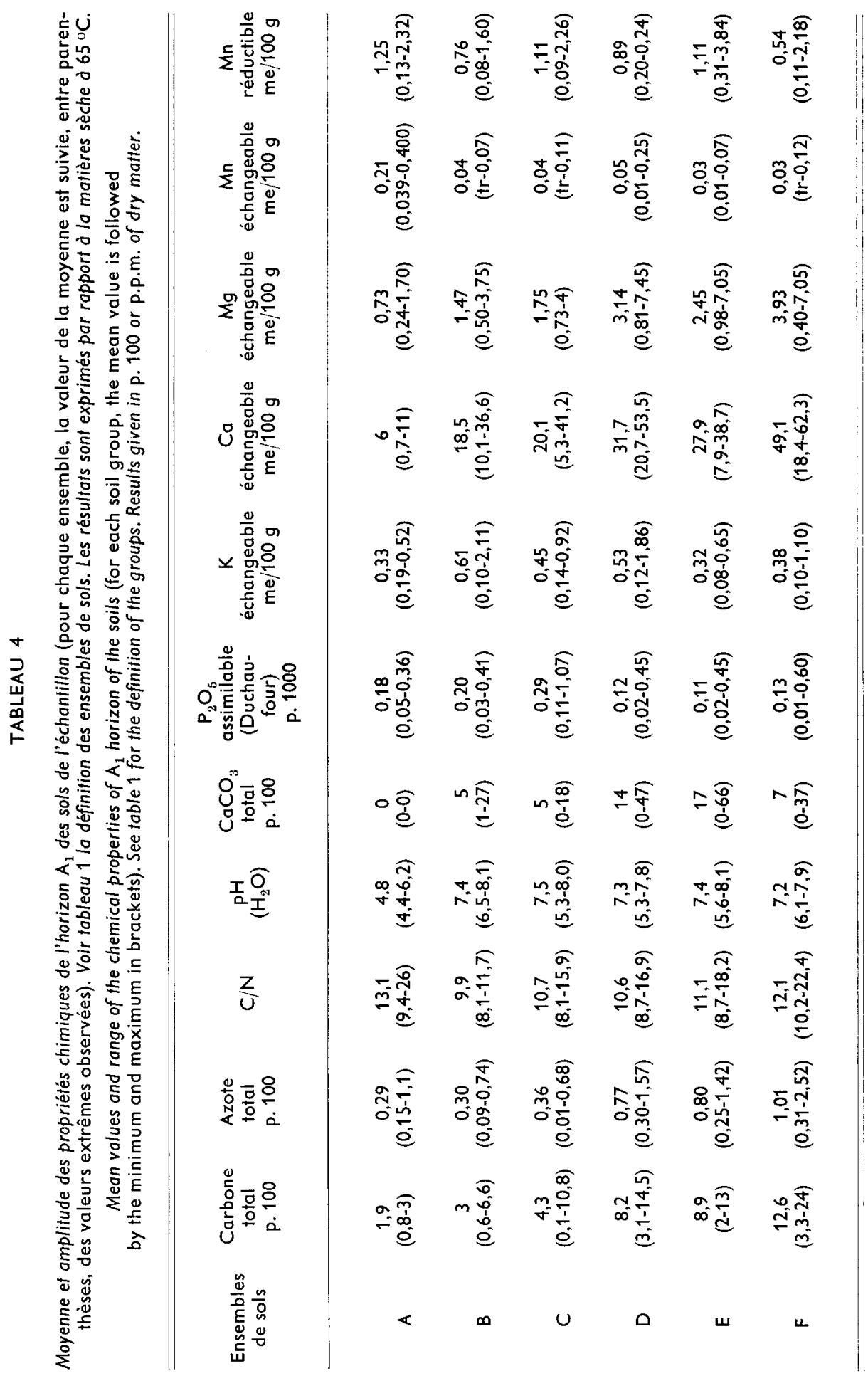


régime hydrique est manifestement le facteur limitant essentiel de la production $(C$ ef $F$ ), et de l'autre les ensembles à bonne production, où le régime hydrique est plus proche de l'optimum ( $D$ et $E$ ). Les coefficients de corrélation ont alors été calculés séparément dans les deux groupes.

Dans le groupe $C+F$ (eau facteur limitant par excès ou par défaut), une seule liaison apparaît encore, la même que dans l'ensemble de l'échantillon : $P$ feuilles- $P$ sol (risque de 1 p. 100) toujours pour « Robusta ».

Par contre, dans le groupe D + E (eau non limitante), un grand nombre de liaisons sont significatives chez ce même clone, alors sur rien n'apparaît avec « |-214 ». Détaillons-les, élément par élément, en se rapportant aux figures 1, 2 et 3.

- Azote : la teneur en $\mathrm{N}$ des feuilles est liée positivement à la production $(r=0,70$, risque de 1 p. 100). Par contre, aucune liaison n'apparaît avec $\mathrm{N}$ total dans l'horizon $A_{1}$, ni dans le rapport $C / N$ dans cet horizon. On sait en effet que ces deux caractères rendent mal compte de la disponibilité réelle en azote assimilable d'un sol : celle-ci varie suivant les périodes de l'année, et ne peut être estimée que par un grand nombre de dosages périodiques de l'azote minéral in sifu $\left(\mathrm{NO}_{3}^{-}\right.$ef $\left.\mathrm{NH}_{4}^{+}\right)$ou par des incubations à l'étuve (Lemée, 1967). Ce genre d'étude serait à entreprendre pour préciser les relations sol-nutrition-production en ce qui concerne l'azote.

- Phosphore : la teneur en $\mathrm{P}$ des feuilles est liée positivement à la production ( $r=0,75$, risque de 0,1 p. 100$)$ et à la teneur en $P$ du sol $(r=0,73$, risque de 0,1 p. 100), De plus ce dernier caractère est directement lié positivement à la production $(r=0,73$. risque de 0,1 p. 100). Ceci montre que le phosphore est, après les conditions hydriques du sol, un facteur limitant la production, et que la méthode Duchaufour-Bonneau utilisée pour doser le phosphore assimilable est très bien adaptée à nos types de sols. Ceci confirme les résultats de Bonneau et al. (1970), qui trouvent que pour la plupart des sols forestiers cette méthode est celle qui concorde le mieux avec la nutrition en phosphore des arbres.

- Soufre : le teneur en $S$ des feuilles est liée négativement à la production $(r=-0,73$, risque de 0,1 p. 100). Cette liaison négative suggère que le soufre serait présent dans les feuilles à des concentrations toxiques, ef non pas, comme l'azote ou le phosphore, à des concentrations inférieures à un optimum par ailleurs inconnu. Nous avons donc cherché ce qui pourrait apporter un tel excès de soufre. La pollution atmosphérique par $\mathrm{SO}_{2}$ aurait pu être une explication, mais nous n'avons trouvé aucune relation entre la teneur des feuilles en soufre et la proximité d'une ville ou d'une usine. Une autre explication pourrait résider dans les horizons organiques profonds de certains sols (tourbe enfouie). Cette matière organique, à pH élevé et à $\mathrm{C} / \mathrm{N}$ bas, est en effet susceptible d'une minéralisation non négligeable malgré l'engorgement presque permanent, et sa fréquente odeur $d^{\prime} \mathrm{H}_{2} \mathrm{~S}$ indique la présence de grandes quantités de sulfures qui peuvent remonter par capillarité avec l'eau de la nappe jusqu'aux racines ; ce soufre pourrait alors être absorbé en quantité excessive sous forme de sulfate après oxydation, ou être la cause d'une toxicité directe sous forme de sulfure. Cette deuxième possibilité est d'ailleurs la plus vraisemblable, car, comme le phosphore, le soufre sous forme de sulfate ne semble pas se prêter à une absorption excessive (Metivier, 1961). Mais la comparaison de la teneur en soufre des feuilles des stations avec ou sans tourbe enfouie ne permet pas de confirmer cette hypothèse. 


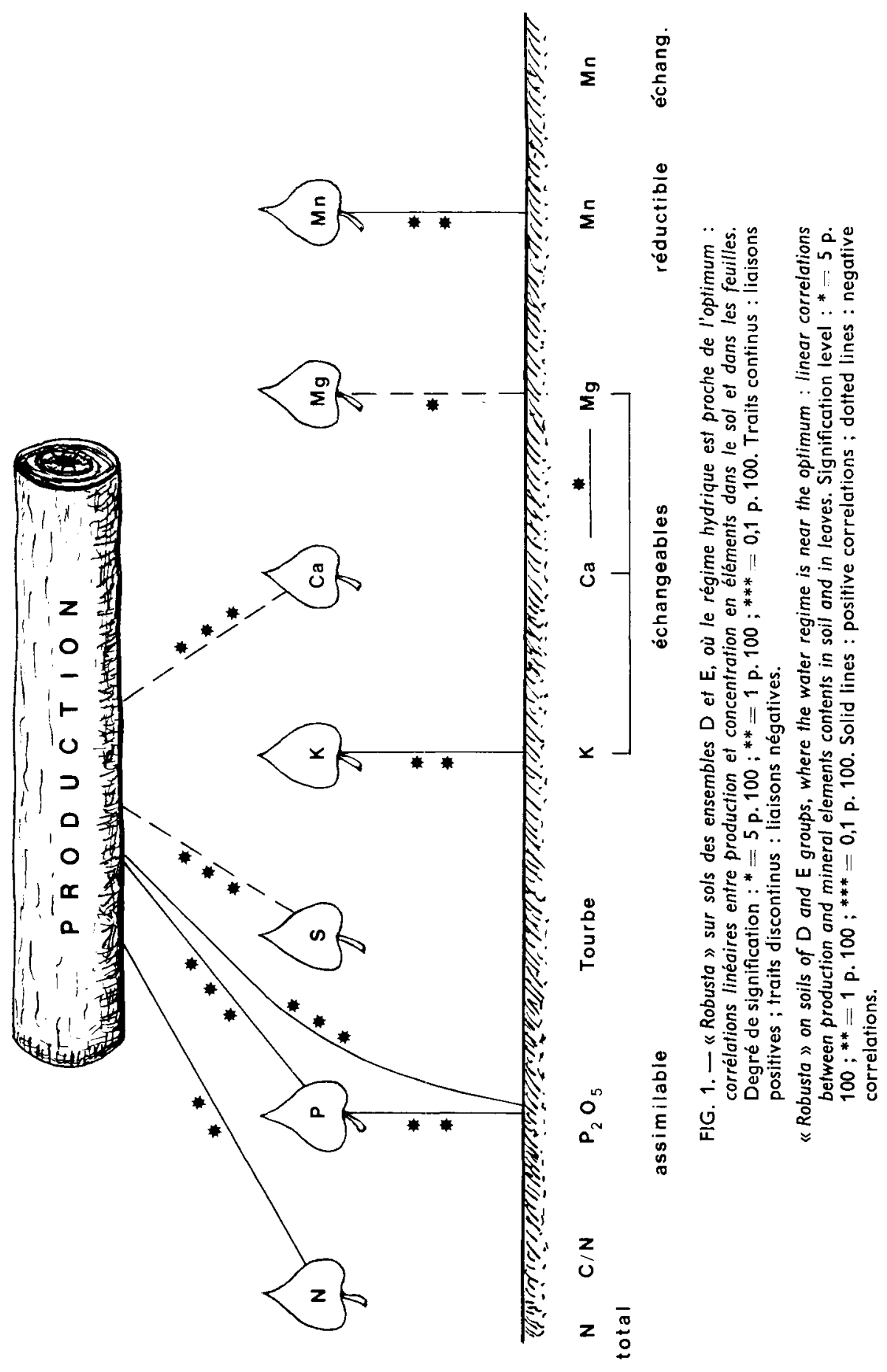



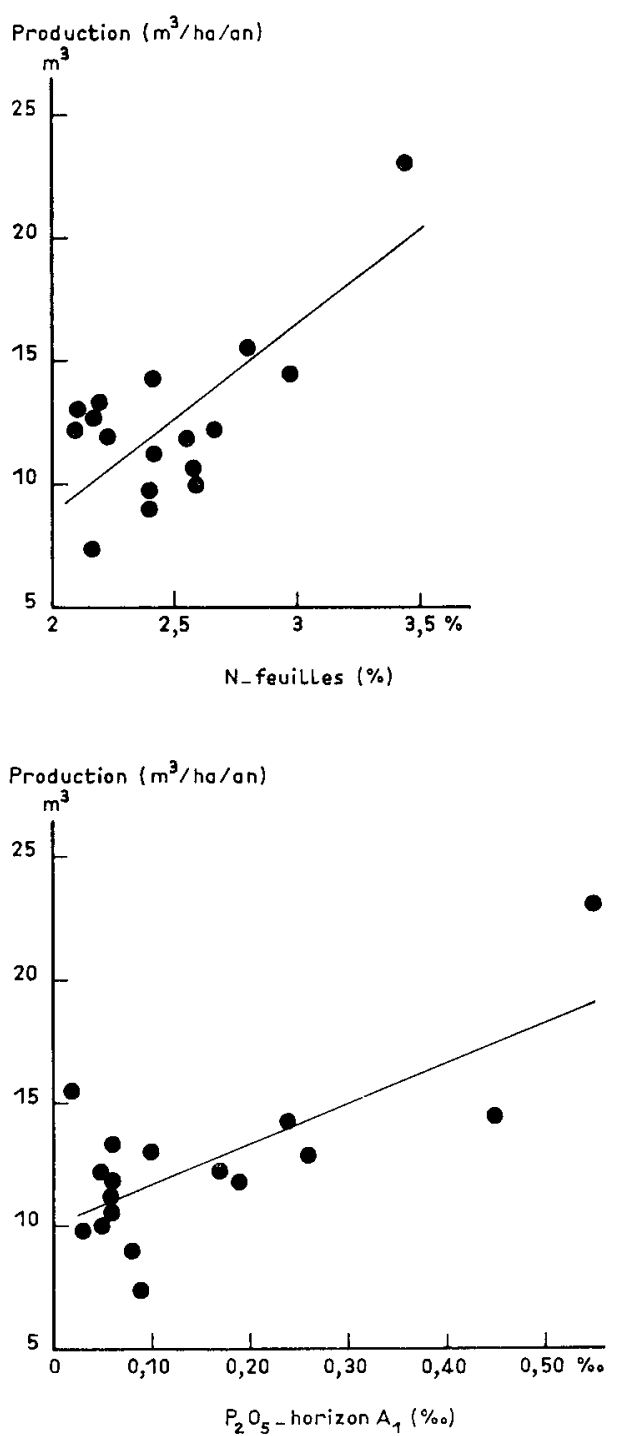
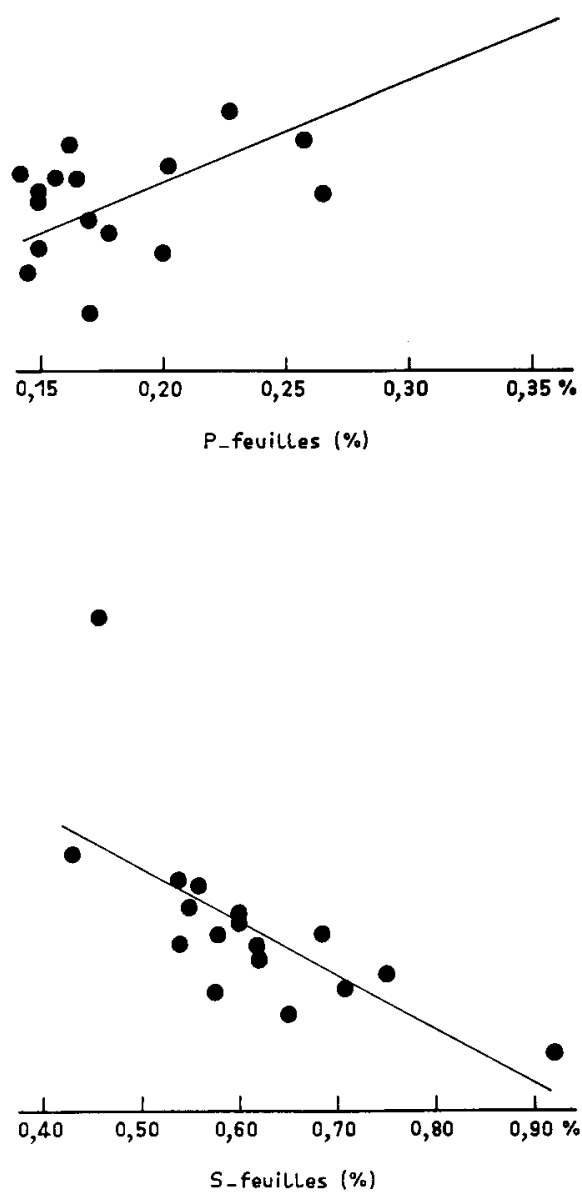

FIG. 2. - «Robusto " sur sols des ensembles D ef $\mathrm{E}$, où le régime hydrique est proche de l'optimum : dispersion des mesures et droite de régression dans le cas des liaisons N-feuilles-production, P-feuillesproduction, $\mathrm{P}_{2} \mathrm{O}_{5}$ sol-production ef $\mathrm{S}$-feuilles production.

"Robusta » on soils of $\mathrm{D}$ and $\mathrm{E}$ groups, where the water regime is near the optimum : dispersion of the measures and regression lines for the following correlations : $N$ leaves-production, $P$ leaves-production, $\mathrm{P}_{2} \mathrm{O}_{5}$ soil-production and $\mathrm{S}$ leaves-production.

Nous avons seulement vu plus haut (tabl. 3) que les valeurs du soufre foliaire étaient plus élevées sur les ensembles de sols où la nappe s'approche le plus de la surface (E et F). Enfin, une dernière possibilité n'est pas à exclure : la liaison négative observée entre la teneur en $\mathrm{S}$ des feuilles ef la production ne traduit peut-être pas une relation de cause à effet, mais traduirait indirectement la relation négative entre la teneur 

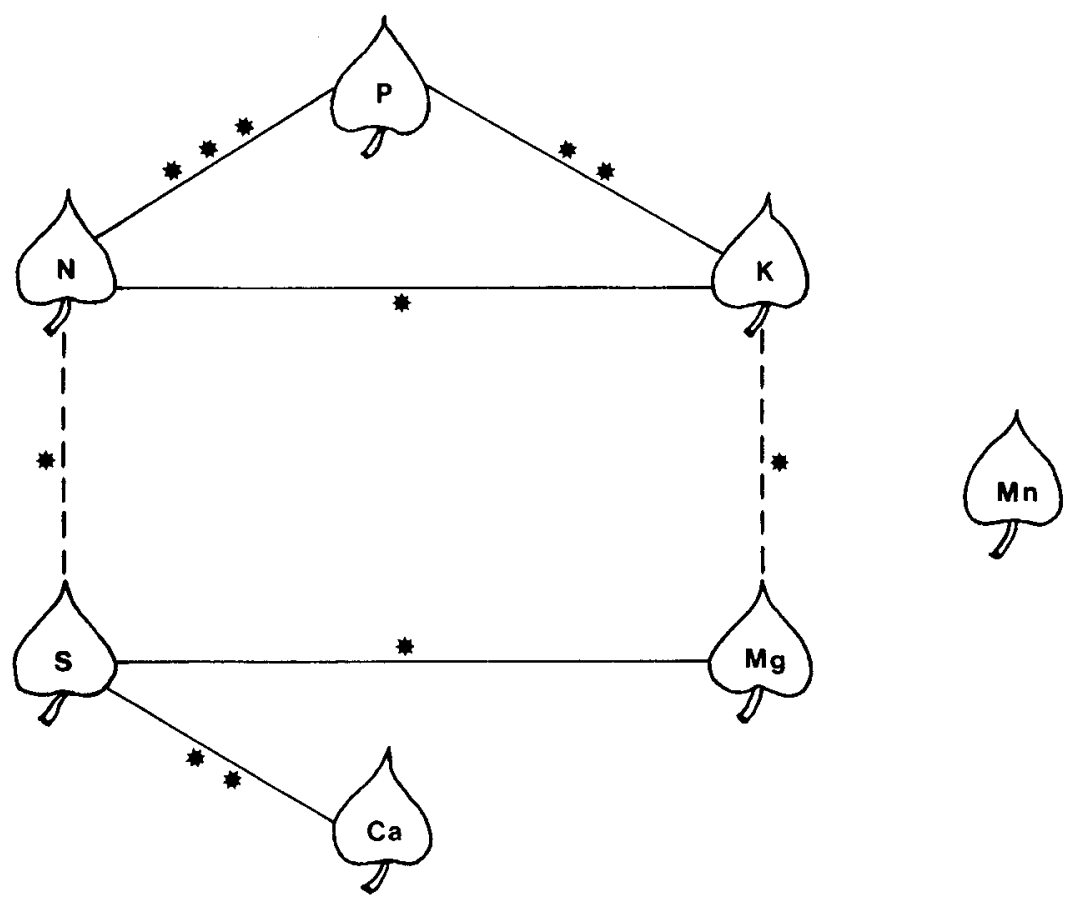

FIG. 3. - «Robusta » sur sols des ensembles D ef E, où le régime hydrique est proche de l'optimum : corrélations linéaires inter-placettes, entre les concentrations foliaires des divers éléments totaux. Degré de signification : $*=5$ p. $100 ; * *=1$ p. $100 ; * * *=0,1$ p. 100 . Traits continus : liaisons positives; traits discontinus : liaisons négatives.

"Robusta 》 on soils of D and E groups, where the water regime is near the optimum : inter-plots linear correlations between foliar confents of the different mineral elements. Signification level : $*=5$ p. 100 ; $* *=1$ p. $100 ;{ }^{* * *}=0,1$ p. 100 . Solid lines : positive correlations ; dotted lines : negative correlations.

en $\mathrm{S}$ des feuilles ef la teneur en $\mathrm{N}$ des feuilles (fig. 3), ce dernier caractère, nous l'avons vu, étant lié positivement à la production. Cette question du soufre reste donc ouverte, ef justifierait une étude particulière.

- Potassium : la teneur en $K$ des feuilles est liée positivement $(r=0,65$, risque de 1 p. 100) à la teneur en $K$ échangeable du sol, mais pas à la production. Cet élément ne semble donc pas être un facteur limitant la croissance des peupliers dans la région étudiée, même si la quantité absorbée dépend des disponibilités du sol. Une telle « consommation de luxe » est d'ailleurs fréquente pour le potassium chez de nombreux végétaux.

- Calcium : comme le soufre, cel élément est seulement lié négativement avec la production ( $r=0,63$, risque de 1 p. 100). Aucune relation n'apparaît ni avec le calcium du sol exprimé en Ca échangeable dans l'horizon $A_{1}$, ni avec la teneur en calcaire de cet horizon. Le calcium est d'ailleurs toujours présent en grande quantité dans nos sols.

- Magnésium : une seule liaison significative apparaît : la teneur en $\mathrm{Mg}$ des feuilles avec $\mathrm{Mg}$ échangeable dans l'horizon $A_{1}$. Cette liaison est cependant négative 
$(r=-0,56$, risque de 5 p. 100). La liaison positive $(r=0,50$, risque de 5 p. 100) qui existe par ailleurs entre $\mathrm{Mg}$ échangeable ef $\mathrm{Ca}$ échangeable dans le sol suggère donc un antagonisme au niveau de l'absorption de ces deux éléments bivalents, au détriment de l'absorption de magnésium. Cet antagonisme n'est cependant jamais la cause d'une carence en magnésium, puisqu'aucune liaison n'apparaît entre la concentration de cet élément dans les feuilles et la production. On remarque aussi sur la figure 2 que $\mathrm{Mg}$ feuilles est négativement lié à $\mathrm{K}$ feuilles (risque de $5 \mathrm{p} .100$ ), ce qui correspond à l'antagonisme plus souvent rencontré entre le magnésium et le potassium chez la plupart des plantes cultivées.

- Manganèse : la nutrition en manganèse ne semble pas affecter la production mais la teneur en $M n$ des feuilles est liée positivement ( $r=0,62$, risque de 1 p. 100) avec $M n$ réductible dans l'horizon $A_{1}$, alors qu'il ne l'est pas avec $M n$ échangeable. Ce résultat ne fait que confirmer l'intérêt de la méthode proposée par Coppenet (1959) pour l'estimation du manganèse assimilable.

Enfin, la figure montre que les corrélations entre éléments au niveau des feuilles permettent de distinguer deux groupes :

$-N$, $P$ et $K$ voient leurs concentrations positivement liées entre elles. Deux de ces éléments $(\mathrm{N}$ et $\mathrm{P})$ sont liés positivement à la production.

$-\mathrm{S}$, Ca ef $\mathrm{Mg}$ sont aussi liés positivement, mais deux d'entre eux ( $\mathrm{S}$ et $\mathrm{Ca}$ ) sont liés négativement à la production.

De plus, des liaisons négatives relient ces deux groupes opposés : N-S et K-Mg. Quant au manganèse, il n'est lié à aucun autre élément.

\section{Conclusion et conséquences pratiques}

Ces résultats montrent que la nutrition minérale ne permet d'expliquer la variabilité de la production à l'intérieur des ensembles de sols précédemment définis que lorsque le régime hydrique n'est plus limitant (soit par défaut, soit par excès d'hydromorphie), et ceci seulement pour « Robusta 》. Le fait que nous n'ayons rien trouvé dans le cas de «l-214» peut être dû à un échantillon trop restreint pour ce clone (dix-neuf placeaux sur sol alluvial, contre vingt-neuf pour « Robusta »). II est en effet peu probable que « $\mathrm{I}-214$ », qui présente un potentiel de croissance supérieur à celui de « Robusta », soit à ce point indifférents aux conditions de nutrition minérale. II est également possible que les attaques répétées de Marssonina brunnea subies par « I-214 》 plusieurs années de suite avant notre étude aient suffisamment perturbé ce clone pour masquer les relations cherchées. Enfin, il ne faut pas oublier que les facteurs microclimatiques n'ont pas été pris en compte, or il se trouve que les peupliers réagissent très fortement à des variations thermiques ou hygrométriques. Dans ces conditions, on peut penser que de tels facteurs peuvent intervenir directement, ou interférer avec les régimes hydriques et masquer les relations recherchées.

Dans le cas de « Robusta » sur sols alluviaux à régime hydrique proche de l'optimum, les premiers éléments limitant la production sont le phosphore et l'azote. Pour une production de $15 \mathrm{~m}^{3} / \mathrm{ha} / \mathrm{an}$ à 20 ans, chiffre que l'on peut considérer comme satisfaisant, les équations de régression montrent que les feuilles doivent contenir 
0,25 p. 100 de phosphore (P) et 2,8 p. 100 d'azote (prélèvement au sommet de l'arbre à la fin du mois d'août). Ces teneurs foliaires sont peu différentes de celles considérées comme optimales dans la littérature, pour divers clones euraméricains (Van der Meiden et al., 1964 ; Fritsche, 1964 qui donnent 2 à 2,5 p. 100 pour l'azote et 0,2 à 0,5 p. 100 pour le phosphore).

Mais, ce qui est plus intéressant dans la pratique, la régression montre que, pour obtenir une production de $15 \mathrm{~m}^{3} /$ ha et par an à 20 ans, l'horizon $A_{1}$ du sol doit contenir 0,3 p. 1000 de $P_{2} \mathrm{O}_{2}$ assimilable (méthode Duchaufour-Bonneau), ce qui est assez élevé par rapport aux sols forestiers habituels, et même par rapport à l'ensemble de notre échantillon (tabl. 4).

Ce résultat, qui souligne les gros besoins en phosphore des peupliers, concorde avec ceux de la plupart des essais de fertilisation sur cette essence dans le nord-est de la France : la fertilisation phosphatée est très souvent la plus efficace, avec les apports d'azote (Barneoud et al., 1970).

Reçu pour publication en août 1979.

\section{Remerciements}

La prospection, effectuée sur un nombre considérable de peupleraies, n'a été possible que grâce au concours des organisations professionnelles de la Forêt Privée et de leurs conseillers forestiers. Nous remercions également les propriétaires-populiculteurs qui ont bien voulu nous autoriser à effectuer les mesures ef prélèvements nécessaires dans leurs plantations.

\section{Summary}

Mineral nutrition and production of «|-214» and «Robusta» poplars grown under traditional silvicultural conditions in north of the «bassin parisien »

The relationships between $\mathrm{N}, \mathrm{P}, \mathrm{S}, \mathrm{K}, \mathrm{Ca}, \mathrm{Mg}$ and $\mathrm{Mn}$ contents in soil and leaves and production of Populus $Y$ euramericana, cv. « Robusta » and « l-214 », have been studied in sixty six plantations in the north of France, managed in a traditionnal way (without any fertilization nor soil tilling).

On hill soils, without any permanent water-table, productivity is ar first related to presence of calcium carbonate in the top horizon of the soil, which reduces both growth and manganese absorbtion.

On alluvial soils in valleys, with a permanent water-table, the first factor limiting production is water regime (lack of water or water excess), determined by the depth or permanent reduction and the depth of temporary reduction.

When water availability reaches near the optimum, the availabilities of nitrogen and phosphorus in soil ( $A_{1}$ horizon) are limiting factors. The available phosphorus content in $A_{1}$ horizon (DuchaufourBonneau method) is very highly significantly related to phosphorus content in leaves and to production. A wood production over 15 cubic meters per hectare and per year at 20 years for « Robusta " (total volume of the stem) corresponds to 0.3 p. 1000 available $P_{2} O_{5}$ in $A_{1}$ horizon and about 0.3 p. 100 total $P$ in the top leaves of the trees at the end of august.

\section{Références bibliographiques}

BARNEOUD C., BONDUELLE P., 1970. Résultats d'essais de fertilisation de peuplier " $1-214$ » en France. Rapport Association Forêt-Cellulose, 137-186.

BONNEAU M., HABIBI H., LE TACON F., 1970. Comparaison de trois méthodes de dosage du phosphore dans divers types de sols forestiers. Bulletin de l'Association Française pour l'Étude du Sol, (2), 19-27. 
COPPENET M., 1959. Le manganèse dans les sols de Bretagne et ses relations avec la croissance des végétaux. Ann. Agron. 2., 155-218.

DEVAUCHELLE R., LEVY G., 1977. Propriétés stationnelles et croissance du Frêne dans l'est de la France ; étude de certaines caractéristiques de cette essence. Ann. Sci. forest., 34 (3), 231-244.

DUCHAUFOUR Ph., BONNEAU M., 1969. Une méthode nouvelle de dosage du phosphore assimilable dans les sols forestiers. Bulletin de l'Association Française pour l'Etude du Sol, no 4.

FRITZSCHE K., 1964. Beziehungen zwischen den Blattspiegelwerten, den Bodennahrstoff gehalt und den Wachstum einiger Schwartz und Balsampappeln - Düngung und Melioration in der Forsfwirtschaft. Deutsche Akademie der Landwirtschaftwissenschaften. Tagungsberichte, 66, 169-192.

GARBAYE J., 1969. Le diagnostic foliaire chez le peuplier. Document interne, C.N.R.F. Nancy.

GARBAYE J., 1972. Influence de la date et de la hauteur du prélèvement sur les résultats de l'analyse foliaire chez deux clones de peuplier. Ann. Sci. forest., 29 (4), 451-463.

GARBAYE J., 1979. Sol et productivité des peupliers « I-214 » et « Robusta » en populiculture traditionnelle dans le nord du bassin parisien. Ann. Sci. forest., 36 (1).

LEMEE G., 1967. Investigation sur la minéralisation de l'azote ef son évolution annuelle dans les humus forestiers in situ. Oecol. Plant., 2, 285-324.

LE TACON F., MILLIER C., 1970. La nutrition minérale de l'Epicéa (Picea excelsa Link.) en sols. carbonatés et en sols décarbonatés. Ann. Sci. forest., 27 (1), 63-88.

LE TACON F., 1974. La nutrition minérale du pin noir d'Autriche (Pinus nigra Arn. ssp. nigricans Host.) sur les plateaux calcaires de l'est de la France. Ann. Sci. forest., 31 (2), 83-95.

METIVIER S., PULBY J. F., 1961. Le soufre en agriculture. Bulletin de l'Association Française pour l'Etude du Sol, nos 9 et 10.

VAN DER MEIDEN H. A., KOLSTER H. W., 1964. Variation de la composition minérale des feuilles de peuplier pendant la période de végétation dans différentes parties de l'arbre. Korte Mededelin, no 61, Wageningen. 